\title{
A meta-analysis of studies related prevalence of depression in Iran
}

Mehdi Mohamadi ${ }^{1}$, Seyed Hossein Mohaqeqi Kamal' ${ }^{2}$ Meroe Vameghi ${ }^{3}$, Hassan Rafiey ${ }^{3}$, Ameneh Setareh Forouzan ${ }^{3}$ Homeira Sajjadi $^{3}$

\author{
Journal of Research \& Health \\ Social Development \& Health Promotion \\ Research Center \\ Vol. 7, No. 1, Jan \& Feb 2017 \\ Pages: 581- 593. \\ DOI: 10.18869/acadpub.jrh.7.1.581 \\ Original Article
}

1. Department of Social Welfare Management, University of Social Welfare and Rehabilitation Sciences, Tehran, Iran

2. Correspondence to: Department of Social Welfare Management, Faculty Member in University of Social Welfare and Rehabilitation Sciences, Tehran, Iran

Email: hosseinmohaqeq@gmail.com

3. Social Determinant of Health Research Center - Faculty Member in University of Social Welfare and Rehabilitation Sciences, Tehran, Iran

Received: 20 Jun 2014

Accepted: 10 Ju1 2014

How to cite this article: Mohamadi M, Mohaqeqi Kamal SH, Vameghi M, Rafiey H, Setareh Forouzan A, Sajjadi H. A metaanalysis of studies related prevalence of depression in Iran. $J$ Research Health2017; 7(1): 581- 593 .

\begin{abstract}
Depression is one of the most important and common mood disorders. This study aimed to present a clear picture of the prevalence of depression in studies conducted in Iran. In this systematic review study, all published relevant studies were searched in credible scientific databases using standard key words. Repeated cases and those outside the time span of this study (1996 to 2011) were excluded. Articles were examined in terms of quality, and irrelevant ones were excluded. Eventually, 145 articles were selected for this systematic review. Fifteen different tools had been used in these articles, mostly standard tools such as Beck's and general health questionnaires. Beck's inventory indicated the prevalence of depression as $52.12 \%$ in students, $37.22 \%$ in the general population, $33.45 \%$ during postmenopausal period and $27.62 \%$ during pregnancy. General health questionnaire revealed the prevalence of depression as $12.02 \%$ in students, $19.46 \%$ in general population. For the older adults, Geriatric Depression Scale (GDS) played an important role. GDS revealed the prevalence of depression in older adults living at home as $57.58 \%$, and in those living in nursing homes $81.85 \%$. Because of its ubiquitous features, Beck's inventory had been used, which showed overall prevalence of depression $42.59 \%$. Considering high prevalence of depression in Iran, greater attention to depression and preventive measures are hugely important.
\end{abstract}

Keywords: Depression, Meta-Analysis, Prevalence

\section{Introduction}

Behavioral and psychological problems show an increasing trend in developing countries, so that in transition to social development and subsequent changes in climate, politics, and economics, people are more exposed to psychological pressures. Accordingly, the issue of psychological health has always been an important health concern in developing countries [1]. Among various psychological health problems created in the process of change, depression is of particular importance. Depression is one of the most important psychiatric disorders and the most common mood disorder, and because of its high prevalence, it is referred to as psychological common cold. Depression is a state in which individuals have at least 5 known symptoms of this disorder for at least two weeks. These symptoms include depressed mood, loss of interest or enjoyment (anhedonia), weight gain or loss, increased or reduced sleep, mental and motor stimulation or retardation, lack of energy, a sense of guilt and worthlessness, and inability to think or concentrate [2]. Recent 
data indicate that approximately 121 million people worldwide suffer depression. Depression is the second leading psychological disorder, and according to the world health organization, it will top the list of psychological diseases in future [3].

Review of various related literature shows that the prevalence of depression varies across different countries depending on the tools used, culture of the society, and sample size [4]. In epidemiological studies in Iran, different methods and tools have been used to assess the prevalence of depression, and different results have been reported. In 1999, Noorbala reported the prevalence of depression in 15 year-old people and older 21\% [5]. Mohammadi et al reported the prevalence as $2.98 \%$ [6]. In a study by Montazeri et al., titled "systematic review of studies on depression in Iran", a limited number of results were reviewed and only studies and prevalence of depression found in them were cited, which ranged from $5.69 \%$ to $73 \%$ [7]. In a systematic review study by Sarokhani et al., overall prevalence of depression among Iranian students was reported $40 \%$ [8], but this study included only articles that had used Beck's Depression Inventory. In their systematic review study, Sadeghi et al. reported overall prevalence of Major Depressive Disorder (MDD) in Iran $4.1 \%$, ranging from $0.3 \%$ to $5.59 \%$ across different provinces [9]. Given the importance of depression and studies conducted in this area reporting different prevalence rates in Iran, and the need for evidence-based policy-making and planning, makes it essential to use existing and purposive future studies. Thus, the present study aims to conduct a structured review of all documents, and summarize data and results obtained to provide a full picture of dimensions of this problem in Iran.

\section{Method}

This is a descriptive systematic review, the best known method, which is actually a secondary analysis of previous studies through structured or systematic search, based on predetermined regulations. Meta-analysis quantifies results from systematic review using statistical methods.
In this study, statistical population consisted of all articles resulting from quantitative study on the prevalence of depression in Iran published from 1996 to 2011. A search was conducted using keywords: depression, dysthymia, melancholia, and mood disorder in SID, Irandoc, Iranmedex, Iran psych, and Magiran, and English keywords; depression, mood disorder, melancholia, dysthymia, and Iran in Medline databank.

In Farsi sources, search was conducted using article title, abstract and keywords. In nondomestic sources, in Pub Med database, using keyword Iran in organizational affiliation section (affiliation) and its combination (And) with keyword depression in title/abstract section, titles and abstracts of all Iranian articles on depression (306 articles) were found. The same method was used for four English keywords, and with the exception of keyword depression, only keyword mood disorder produced results (28 articles).

Study inclusion criteria included studies conducted on the prevalence of depression in Iranian population in the recent 15 years (1996-2011). Studies conducted on non-human subjects, and those tool or methodology with problems were excluded.

Given the above criteria, ultimately 4077 articles were assessed. In the first screening stage, repeated cases and those outside the 15-year time span were excluded (it should be noted that articles extracted from a reference article were also excluded as repeats, and reference article was included only), and thus 1234 articles remained. In the second screening stage, 753 articles were excluded according to the criteria of relevance to study subject and exclusion of repeated articles in English, and the remainder (481 articles) entered qualitative assessment stage. Qualitative assessment of articles was performed by integration of qualitative assessment checklists, including one by Vameghi (combining existing guidelines with a few innovative questions) [10], and checklists of Critical Appraisal Skills Program (CASP) site. At this stage, articles obtained were assessed by two team members, and if no 
consensus was reached, they were referred to a third member. Eventually, a total of 70 articles were excluded for lack of minimum acceptable quality, and of the remaining 411 articles, 145 relevant articles to "prevalence of depression" entered systematic review stage.

The results from each of the final articles were entered into data input form as data collection tool, in preparation for meta-analysis. To assess possibility of meta-analysis of data, the statistical evaluations are not enough to identify homogeneity of data [11], so the observation method was used as well as Chi-square test. Chi-square test results suggested no significant difference between data from various studies, and thus meta-analysis could be performed on these data. Moreover, observation showed that data could be combined. Next, in each study, equivalent sample size was determined according to its weight, and mean weight of each study found. Articles that had used only one tool were combined and their mean weight was extracted.

\section{Results}

Following stages of search, screening, and qualitative assessment of studies, meta-analysis was conducted on 145 articles (Figure 1).

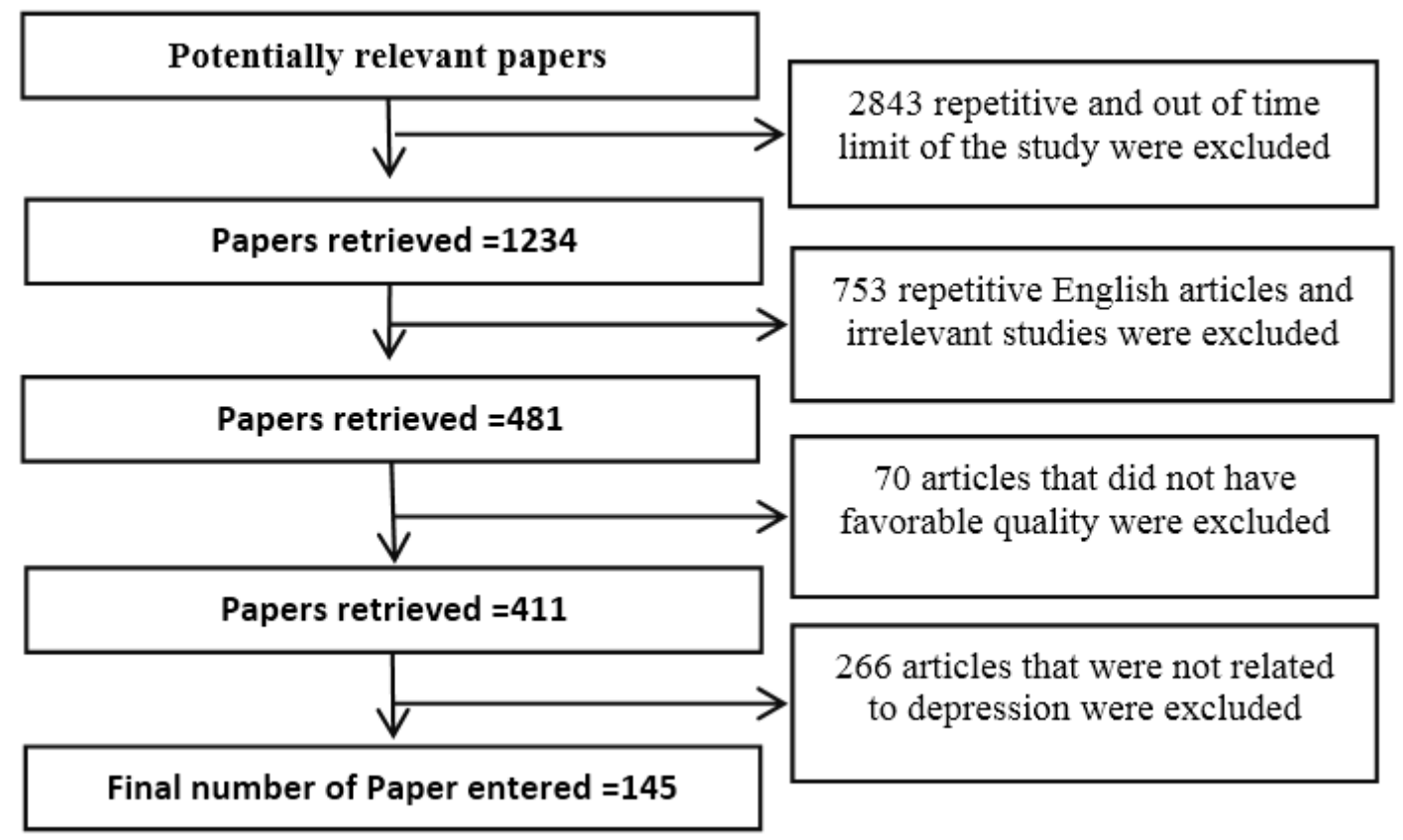

Figure 1 Stages of inclusion and exclusion of studies into final meta-analysis

These studies included a total sample size of 132001 people. Of these studies, 139 had been conducted in urban areas (or in combined urban and rural areas), 4 in rural and 2 in tribal areas. Investigation showed that 15 different tools had been used in these studies, namely Beck Depression Inventory, Edinburgh Postnatal Depression Scale, Geriatrics Depression Scale (GDS), Children Depression Inventory (CDI), Self- Rating Depression Scale of Zung, Standardized Depression Scale (DVYSS), Center for Epidemiology Studies-Depression Child (CES-DC), and Depression, Anxiety and Stress Scale (DASS21). General Health
Questionnaire (GHQ) and Duke's Health Screening questionnaire were used in assessment of general health, and Schedule for Affective Disorders and Schizophrenia (SADS), Symptoms Check List-90 (SCL90), clinical interviews, Rutter children's behaviour questionnaire and Kiddie Schedule for Affective Disorders and Schizophrenia (K-SADS) were used to measure psychiatric disorders. Each measured the concept of depression in its own way. In the present study, only data relating to depression in these studies were used in process of analysis. Of these tools, 6 that appeared in most studies were used in 
this systematic review, including BDI, GHQ, SCL-90, Edinburgh, GDS, and CDI. Articles obtained were examined in 4 general classes of school pupils (31 articles), university students (39 articles), general public (21 articles), and the older adults (15 articles), and also a secondary class called women's depression (40 articles). One of the articles concurrently addressed pupils' and women's depression [12], and was repeated in both groups, which made the total number of articles
146 instead of 145. Next, studies concerned with the prevalence of depression in different groups and types of tools used were assessed, and finally, overall prevalence of depression was investigated using more usual tools.

1- Prevalence of depression in pupils: A total of 31 articles were found on depression in pupils, most of which had addressed depression among high school students. Details of these studies are presented in Table 1.

Table 1 Summary of studies associated with pupils'depression

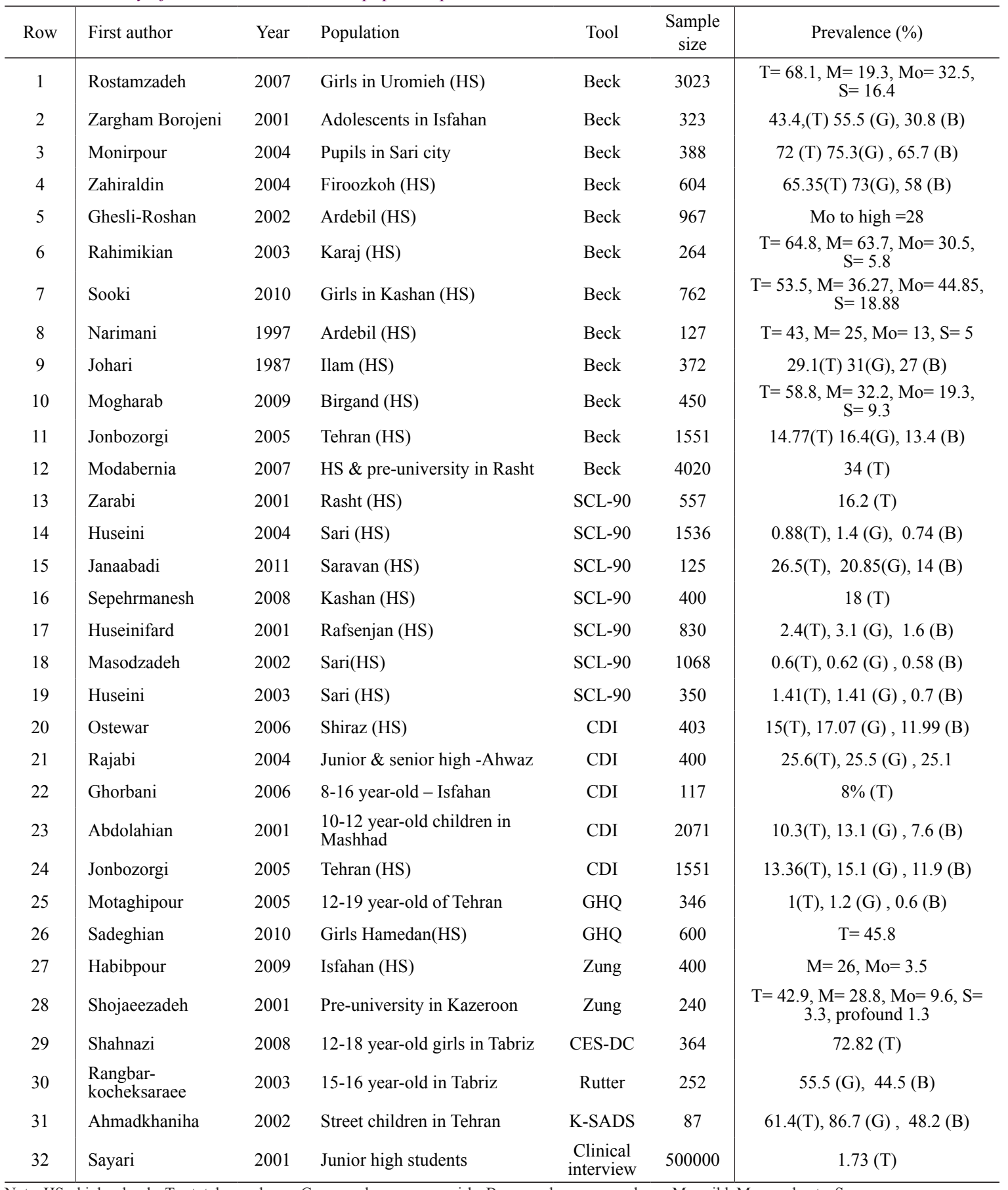

Note. $\mathrm{HS}=$ high schools, $\mathrm{T}=$ total prevalence, $\mathrm{G}=$ prevalence among girls, $\mathrm{B}=$ prevalence among boys, $\mathrm{M}=$ mild, $\mathrm{Mo}=$ moderate, $\mathrm{S}=$ severe 
One of the articles in this category had concurrently used both BDI and CDI tools, which explains the 32 rows instead of 31 in Table 1.

According to details presented in Table 1 relating to depression in school pupils, meta-analysis of articles resulted in three questionnaires of BDI, SCL-90, and CDI, which appeared in sufficient number of articles. Twelve articles used BDI to assess depression among school pupils with overall sample size 12851 pupils. Using mean weight of studies, prevalence of depression in school pupils using BDI was found $43.55 \%$. Among these articles, 8 had assessed prevalence of depression in different sexes, and following calculation of mean weight, prevalence of depression was found $57.69 \%$ in girls and $30.09 \%$ in boys.

Furthermore, in depression scales only in boys measures severity of depression, and in these 12 articles, only 6 reported severity of depression in pupils as follows: $25.95 \%$ had mild depression, $31.58 \%$ moderate depression, and $14.92 \%$ had severe depression.

Of the 7 articles that used SCL-90 to assess pupils' depression, 5 were processed in this study [13-17], and the other two were excluded, since they did not provide an estimate of prevalence of depression. The overall sample size in these 5 articles was 2262 pupils, and prevalence of depression in pupils using this tool was found $15.87 \%$.

Overall sample size of 5 articles that used CDI to assess pupils' depression was 4542 pupils, and mean weight of these articles showed a $13.05 \%$ prevalence of depression in pupils. Four articles reported prevalence of depression according to gender, with prevalence of $15.32 \%$ in girls and $11.05 \%$ in boys.

2- Prevalence of depression in university students: A total of 39 articles were found to have addressed prevalence of depression among university students. Details of these articles are presented in Table 2 .

According to Table 2, two questionnaires of BDI and GHQ (which had been used in sufficient number of articles) were used in calculation of mean weight. Of the 25 articles that used BDI to assess students' depression, 24 were used in synthesis and mean weight calculation. Overall sample size of these articles was 7258 students, and mean weight of prevalence of depression in students using BDI was found 52.12. Of these studies, 11 reported prevalence of depression in female students $(48.35 \%)$ and 10 in male students $(50.48 \%)$. Using this tool, 19 articles reported severity of depression mild to moderate, and 20 reported severe depression in students. Accordingly, in the present study, 32.71\% were found with mild depression, $15.03 \%$ with moderate, and $8.14 \%$ with severe depression.

According to the search conducted, 10 articles used GHQ to assess prevalence of depression in students, of which 7 were used in this study in calculation of mean weight and overall prevalence. Overall sample size of these studies was 1380 students. Thus, prevalence of depression in students, using this tool, was found $12.02 \%$.

3- Prevalence of depression in general population: In assessment of prevalence of depression in general public, studies that completed questionnaire by interviewing people at home were used. Accordingly, after a critical review, 21 articles entered final synthesis. Details of these studies are presented in Table 3.

Among studies on prevalence of depression among general population, one article with sample size of 25180 people had used SADS, and found prevalence of depression $2.98 \%$ across Iran. In relation to prevalence of depression in different sexes, prevalence was reported $4.38 \%$ in women and $1.59 \%$ in men. It should be noted that 18 other articles (including data on prevalence of depression in provinces) that had been extracted from this article were excluded from this study to prevent repeat of data analysis in systematic review. 
Table 2 Summary of articles relating to depression in students

\begin{tabular}{|c|c|c|c|c|c|c|}
\hline Row & First author & Year & $\begin{array}{l}\text { Population } \\
\text { (University) }\end{array}$ & Tool & $\begin{array}{l}\text { Sample } \\
\text { size }\end{array}$ & Prevalence $(\%)$ \\
\hline 1 & Tavakolizadeh & 2001 & Gonabad (UMS) & Beck & 291 & $62.5(\mathrm{~T}), 43.8(\mathrm{G}), 57.6(\mathrm{~B})$ \\
\hline 2 & Dadkhah & 2009 & Ardebil (UMS) & Beck & 409 & $50.8(\mathrm{~T})$ \\
\hline 3 & Abdolahian & 1999 & Ferdowsi-Mashhad & Beck & 420 & $\mathrm{~T}=66.7, \mathrm{M}=44.3, \mathrm{Mo}=16.4, \mathrm{~S}=6$ \\
\hline 4 & $\begin{array}{l}\text { Hashemi- } \\
\text { Mahmoodabad }\end{array}$ & 2001 & Yasuj & Beck & 464 & $\begin{array}{c}\mathrm{T}=63.8, \mathrm{M}=28.2, \mathrm{Mo}=11.6 \\
\mathrm{~S}=20.7, \mathrm{P}=3.2\end{array}$ \\
\hline 5 & Mansoor Ghanaee & 2007 & $\begin{array}{l}\text { Lahijan and } \\
\text { Langrood }\end{array}$ & Beck & 110 & $\begin{array}{c}\mathrm{T}=61.8, \mathrm{M}=37.3, \mathrm{Mo}=14.5, \\
\mathrm{~S}=10\end{array}$ \\
\hline 6 & Sharifi & 2000 & Kashan(UMS) & Beck & 307 & $35.8(\mathrm{~T}), 34.4(\mathrm{G}), 38.8(\mathrm{~B})$ \\
\hline 7 & Salehi & 2000 & Arak (UMS) & Beck & 153 & $22.1(\mathrm{~T})$ \\
\hline 8 & Eslami & 2002 & Gorgan (UMS) & Beck & 238 & $10.56(\mathrm{~T})$ \\
\hline 9 & Rashidi-zavieh & 2000 & Zanjan (UMS) & Beck & 148 & $48.6(\mathrm{~T})$ \\
\hline 10 & Ghaneie-Motlaq & 2003 & Mashhad (UMS) & Beck & 254 & $37(\mathrm{~T}), 36.6(\mathrm{G}), 35.5(\mathrm{~B})$ \\
\hline 11 & Karimi-zarachi & 2003 & Medicine & Beck & 197 & $28.9(\mathrm{~T})$ \\
\hline 12 & Amani & 2004 & Ardebil (UMS) & Beck & 324 & $\begin{array}{c}\mathrm{T}=57.4, \mathrm{M}=18.5 \text {, counseling } \\
\text { required } 10.2 \text {, relatively } 16.4, \mathrm{~S}= \\
8.2 \text {, profound } 4\end{array}$ \\
\hline 13 & Forotani & 2005 & $\begin{array}{l}\text { Institute of higher } \\
\text { education in Larr }\end{array}$ & Beck & 134 & $42.5(\mathrm{~T}), 41.2(\mathrm{G}), 45.9(\mathrm{~B})$ \\
\hline 14 & Ghasemi & 2008 & $\begin{array}{l}\text { Mashahd- Azad } \\
\text { (UMS) }\end{array}$ & Beck & 780 & $28.6(\mathrm{~T}), 31.3(\mathrm{G}), 18.7(\mathrm{~B})$ \\
\hline 15 & $\begin{array}{l}\text { Baghiani- } \\
\text { Moghadam }\end{array}$ & 2006 & Yazd (UMS) & Beck & 185 & $\begin{array}{c}\mathrm{T}=52.8, \mathrm{M}=24.4, \mathrm{Mo}=22.4, \\
\mathrm{~S}=6.2\end{array}$ \\
\hline 16 & Hashemi & 2001 & Yasuj (UMS) & Beck & 421 & $69.7(\mathrm{~T}), 65(\mathrm{G}), 73(\mathrm{~B})$ \\
\hline 17 & Abedini & 2007 & Hormozgan (UMS) & Beck & 190 & $54.75(\mathrm{~T})$ \\
\hline 18 & Ilderabadi & 2004 & Zabol (UMS) & Beck & 175 & $64.3(\mathrm{~T}), 64.4(\mathrm{G}), 60.9(\mathrm{~B})$ \\
\hline 19 & Zohor & 2001 & Kerman (UMS) & Beck & 346 & $61(\mathrm{~T})$ \\
\hline 20 & Hashemi & 2004 & Yasuj- Azad & Beck & 452 & $62(\mathrm{~T}), 71.8(\mathrm{G}), 75(\mathrm{~B})$ \\
\hline 21 & Fakhari & 2001 & Tabriz & Beck & 600 & $50(\mathrm{~T}), 41.5(\mathrm{G}), 38.14(\mathrm{~B})$ \\
\hline 22 & Najafipour & 2008 & Jahroom (UMS) & Beck & 137 & $45.4(\mathrm{~T}), 48.5(\mathrm{G}), 45.9(\mathrm{~B})$ \\
\hline 23 & Mohammadzadeh & 2011 & Ilam & Beck & 381 & $76.1(\mathrm{~T})$ \\
\hline 24 & Jalilian & 2011 & Hamedan (UMS) & Beck & 235 & $\begin{array}{c}\mathrm{T}=37, \mathrm{M}=18.7, \mathrm{Mo}=16.2, \mathrm{~S}= \\
2.1\end{array}$ \\
\hline 25 & Ahmari & 2009 & Qum (UMS) & Beck & 250 & $\begin{array}{c}\mathrm{T}=55.2, \mathrm{M}=37.2, \mathrm{Mo}=14.83 \\
\mathrm{~S}=3.2\end{array}$ \\
\hline 26 & Karami & 2001 & Zanjan (UMS) & GHQ & 395 & $\mathrm{~T}=26.4, \mathrm{Mo}=21.8, \mathrm{~S}=4.6$ \\
\hline 27 & Rezaee & 2006 & Nursing Amol & GHQ & 85 & $4.13(\mathrm{~T})$ \\
\hline 28 & Ansari & 2007 & Zahedan (UMS) & GHQ & 240 & $17.1(\mathrm{~T})$ \\
\hline 29 & Ahmadi & 2007 & Shahrood- Azad & GHQ & 360 & $\begin{array}{c}\text { Nursing }=17.8, \text { non, nursing }=28.8 \\
(\text { Mo \& S })\end{array}$ \\
\hline 30 & Saki & 2002 & Ilam (UMS) & GHQ & 175 & $3.7(\mathrm{~T}), 3.3(\mathrm{G}), 4.9(\mathrm{~B})$ \\
\hline 31 & Tavakolizadeh & 2002 & Gonabad (UMS) & GHQ & 140 & $2.5(\mathrm{~T})$ \\
\hline 32 & Adham & 2008 & Ardebil (UMS) & GHQ & 352 & $S=2$ \\
\hline 33 & Dalir & 2003 & Mashhad (UMS) & GHQ & 209 & $2.77(\mathrm{~T})$ \\
\hline 34 & Akasheh & 2000 & Kashan (UMS) & GHQ & 253 & Major 8.7, double 3.4 \\
\hline 35 & Tavakolizadeh & 2010 & Gonabad (UMS) & GHQ & 136 & $2.07(\mathrm{~T})$ \\
\hline 36 & Farhadi & 2008 & Lorestan (UMS) & SCL-90 & 174 & $9.7(\mathrm{G}), 11.8(\mathrm{~B})$ \\
\hline 37 & Huseini & 2000 & Mazandaran (UMS) & SCL-90 & 279 & $1.03(\mathrm{~T}), 1.16(\mathrm{G}), 0.82(\mathrm{~B})$ \\
\hline 38 & Parvizifard & 2006 & Kermanshah (UMS) & $\begin{array}{c}\text { Clinical } \\
\text { interview }\end{array}$ & 423 & $5.67(\mathrm{~T}), 6.37(\mathrm{G}), 3.67(\mathrm{~B})$ \\
\hline 39 & Rezaee & 2007 & Tarbiat Modaress & DASS21 & 223 & $\mathrm{~T}=51.6, \mathrm{Mo}=41.3, \mathrm{~S}=10.3$ \\
\hline
\end{tabular}

Note. $\mathrm{UMS}=$ University of medical science $\mathrm{T}=$ total prevalence, $\mathrm{G}=$ prevalence among girls, $\mathrm{B}=$ prevalence among boys, $\mathrm{M}=$ mild, $M o=$ moderate, $\mathrm{S}=$ severe 
Meta-analysis of studies related prevalence

Table 3 Summary of articles relating to prevalence of depression in general public

\begin{tabular}{|c|c|c|c|c|c|c|}
\hline Row & First author & Year & Population & Tool & $\begin{array}{l}\text { Sample } \\
\text { size }\end{array}$ & Prevalence (\%) \\
\hline 1 & Mohammadi & 2003 & $\operatorname{Iran}(\mathrm{U}, \mathrm{R})$ & SADS & 25180 & $7.98(\mathrm{~T})-4.38(\mathrm{~W})-1.59(\mathrm{M})$ \\
\hline 2 & Kheirabadi & 2000 & Kamyaran (U) & Beck & 1401 & $69.5(\mathrm{~T})-71.9(\mathrm{~W})-67(\mathrm{M})$ \\
\hline 3 & Khoshhal & 2004 & Isfahan & Beck & 800 & $50(\mathrm{~T})$ \\
\hline 4 & Nazari & 2007 & Khoramabad & Beck & 2740 & $33.4(\mathrm{~T})-34.3(\mathrm{~W})-32.4(\mathrm{M})$ \\
\hline 5 & Kaviani & 2000 & Tehran & Beck & 1052 & $11.2(\mathrm{~T})-13.8(\mathrm{~W})-8.8(\mathrm{M})$ \\
\hline 6 & Bakhshi & 2007 & Rafsenjan & Beck & 600 & $26.7(\mathrm{~T})-27.7(\mathrm{~W})-25.7(\mathrm{M})$ \\
\hline 7 & Ragabizadeh & 2004 & Mahan-Kerman & Beck & 737 & $59.17(\mathrm{~T})$ \\
\hline 8 & Ahmadi & 2009 & Isfahan (U, R) & Beck & 403 & $23.1(\mathrm{~T})$ \\
\hline 9 & Nazari & 2001 & Tehran & Beck & 1191 & $22.5(\mathrm{~T})-29.2(\mathrm{~W})-16(\mathrm{M})$ \\
\hline 10 & Mohammadi & 2007 & Qashqai nomads & Beck & 181 & $35.89(\mathrm{~T})$ \\
\hline 11 & Ahmadi & 2008 & $\begin{array}{l}\text { Bakhtiari } \\
\text { nomads }\end{array}$ & Beck & 400 & $29.6(\mathrm{~T})$ \\
\hline 12 & Motaqipour & 2005 & $\begin{array}{l}\text { District } \\
\text { 13-Tehran }\end{array}$ & GHQ & 927 & $0.75(\mathrm{~T})-0.9(\mathrm{~W})-0.6(\mathrm{M})$ \\
\hline 13 & Parvaresh & 2011 & Kerman & GHQ & 1527 & $6.81(\mathrm{~T})$ \\
\hline 14 & Noorbala & 1999 & Iran & GHQ & 35014 & $21(\mathrm{~T})-25.9(\mathrm{~W})-14.9(\mathrm{M})$ \\
\hline 15 & Omidi & 2008 & Natanz (U) & GHQ & 650 & $3.4(\mathrm{~T})-5.5(\mathrm{~W})-1.2(\mathrm{M})$ \\
\hline 16 & $\begin{array}{l}\text { Shams- } \\
\text { alizadeh }\end{array}$ & 2001 & Savugbelaq (R) & GHQ & 640 & $11.3(\mathrm{~T})-15.7(\mathrm{~W})-5.4(\mathrm{M})$ \\
\hline 17 & Ahmadvand & 2010 & Kashan & GHQ & 1800 & $9(\mathrm{~T})$ \\
\hline 18 & Khosravi & 2002 & Borogen (U) & SCL-90 & 450 & $11.76(\mathrm{~T})-15.76(\mathrm{~W})-6.3(\mathrm{M})$ \\
\hline 19 & Hassanshahi & 2003 & Arsenjan (U) & SCL-90 & 650 & $7.73(\mathrm{~T})-5.58(\mathrm{~W})-10.52(\mathrm{M})$ \\
\hline 20 & Fakhari & 2003 & $\begin{array}{l}\text { Tabriz- North- } \\
\text { East }\end{array}$ & Duke & 2076 & $18.95(\mathrm{~W})-6.84(\mathrm{M})$ \\
\hline 21 & Sadeghi & 2000 & Kermanshah (U) & $\begin{array}{c}\text { Clinical } \\
\text { interview }\end{array}$ & 501 & $1.6(\mathrm{~T})-1.85(\mathrm{~W})-1.3(\mathrm{M})$ \\
\hline
\end{tabular}

Note. $\mathrm{U}=$ Urban, $\mathrm{R}=$ Rural, $\mathrm{T}=$ total prevalence, $\mathrm{W}=$ prevalence among women, $\mathrm{M}=$ prevalence among Men

Ten studies had used BDI, and their overall sample size was 9504 people. Using mean weight of these studies, prevalence of depression in general population of Iran using BDI was $37.32 \%$. Of these 10 studies, 6 reported prevalence of depression in different sexes, so that prevalence of depression in women was found $37.71 \%$ and in men $31.86 \%$.

Of the 6 studies that used GHQ in assessment of depression in general public, one did not provide an estimate of prevalence and was excluded
[18], and mean weight was calculated for the remaining 5 studies, and thus prevalence of depression in general population was found $19.46 \%$. Overall sample size of these studies was 39631 people.

4- Prevalence of depression in the older adults and women: Following a critical review, 15 studies on prevalence of depression in the older adults and 40 studies on prevalence of depression in the women entered final analysis of the present study (Table 4). 
Table 4 Summary of studies on prevalence of depression in the older adults and women

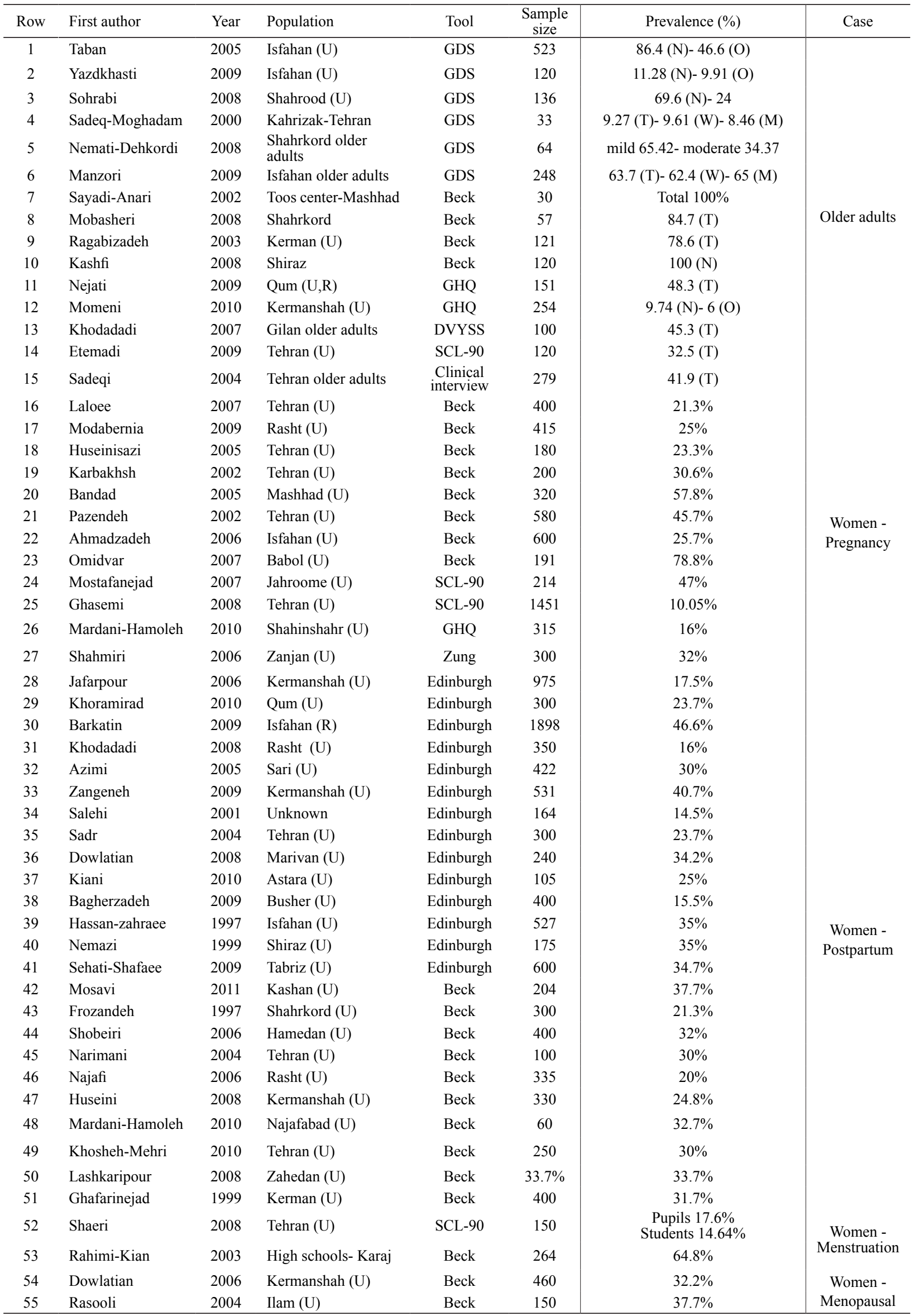

Note. $\mathrm{U}=$ Urban, $\mathrm{R}=$ Rural, $\mathrm{N}=$ Nursing home, $\mathrm{O}=$ own home, $\mathrm{T}=$ total prevalence, $\mathrm{W}=$ prevalence among women, $\mathrm{M}=$ prevalence among $\mathrm{Men}$ 
BDI and GDS tools had sufficient appearance in articles for obtaining mean weight of prevalence of depression in older adults. Based on these studies, prevalence of depression in older adults living at home and older adults living in nursing homes can be separately calculated. Among studies that used GDS tool, only two addressed depression in older adults living in nursing homes. Total sample size of these studies amounted to 170 people. Thus, mean weight of prevalence of depression in older adults living in nursing homes was found $81.85 \%$. Three studies investigated prevalence of depression in older adults living at home. Total sample size of these studies was 761 people. Thus, mean weight and prevalence of depression in older adults living at home was found $57.58 \%$.

Four articles used BDI to find prevalence of depression in older adults, of these three focused on older adults in nursing homes, and one on those living at home. Thus, prevalence of depression in older adults in nursing homes is reported here only. Total sample size of these three articles was 207 people. Therefore, mean weight of prevalence of depression in older adults in nursing homes was found $95.64 \%$. It should be added that overall sample size of studies on prevalence of depression in older adults was 1138 people, but due to absence of sufficient data, no estimate could be found for prevalence of depression in older adults in different sexes.

Depression in women categorized in five case includes depression during pregnancy, postpartum, menstruation or premenstrual syndrome, and menopausal. Of the 12 articles on prevalence of depression during pregnancy, 8 had used BDI, and were used in final synthesis here. Overall sample size of these studies was 2886 women, and mean weight was found $27.62 \%$.

Of the 24 articles used in finding mean weight of studies on postpartum depression, 14 had used Edinburgh scale and 10 had used BDI. Mean weight of the first 14 with overall sample size of 6987 women, was found $32.52 \%$, and mean weight of the next 10 with overall sample size of 2679 women was found $28.66 \%$. Total sample size of studies on postpartum depression was 9666 women.

Since the two studies on menstruation depression had used different tools, metaanalysis could not be performed on their results. However, two studies on menopausal depression had both used BDI. Total sample size in these studies was 610 women. Thus, mean weight was found $33.45 \%$. Overall sample size of studies on women's depression used in final synthesis was 13172 women.

5- Overall prevalence of depression: of the 15 different tools used in studies to find prevalence of different dimensions of depression, 7 appeared in large number of studies, thus meta-analysis could be performed on them. Out of these tools, SADS had only been used to find depression in general public, and CDI, GDS and Edinburgh were used specifically in adolescents, older adults and postpartum depression respectively and prevalence in each group was discussed above. Hence, only three tools of BDI, GHQ, and SCL-90 had been used in different groups and were able to undergo meta-analysis. Summary of studies that used these tools is shown in Table 5 (studies that did not provide estimates of prevalence were not reported).

Table 5 shows that the majority of studies had used BDI, which was the only common tool in all groups. Hence, BDI can be considered the basis in calculation of overall prevalence of depression. Other than the two studies that did not provide an estimate of prevalence, 70 studies entered systematic review, and overall prevalence of depression with this tool was found $42.59 \%$ (it should be noted that one article had used BDI in concurrent assessment of depression in students and women, and was repeated in both groups, making total number of articles 71. Moreover, one article had used GHQ in concurrent assessment of older adults' depression at home and in nursing homes, making the total number of articles using GHQ scale 18 instead of 17). 
Table 5 Summary of studies on overall prevalence of depression according to different tools used

\begin{tabular}{|c|c|c|c|c|c|c|c|c|c|c|c|}
\hline \multirow{3}{*}{ ¿ } & \multirow{3}{*}{ Indicators } & \multicolumn{9}{|c|}{ Groups } & \multirow{3}{*}{ Total } \\
\hline & & \multirow[b]{2}{*}{ Pupils } & \multirow[b]{2}{*}{ Students } & \multirow{2}{*}{$\begin{array}{l}\text { General } \\
\text { population }\end{array}$} & \multicolumn{2}{|c|}{ Older adults } & \multicolumn{4}{|c|}{ Women } & \\
\hline & & & & & Home & $\begin{array}{l}\text { Nursing } \\
\text { home }\end{array}$ & A & $\mathrm{B}$ & $\mathrm{C}$ & $\mathrm{D}$ & \\
\hline \multirow{3}{*}{$\bar{\partial}$} & $\begin{array}{l}\text { Number of } \\
\text { studies }\end{array}$ & 12 & 24 & 10 & 1 & 3 & 8 & 10 & 2 & 1 & 70 \\
\hline & Sample size & 12851 & 7258 & 9504 & 1212 & 207 & 2886 & 2679 & 610 & 264 & 37207 \\
\hline & Prevalence $\%$ & 43.55 & 52.12 & 37.32 & 78.60 & 95.64 & 27.62 & 28.66 & 33.45 & 64.8 & 42.59 \\
\hline \multirow{3}{*}{ 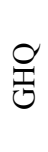 } & $\begin{array}{l}\text { Number of } \\
\text { studies }\end{array}$ & 2 & 7 & 5 & 1 & 2 & 1 & - & - & - & 17 \\
\hline & Sample size & 946 & 1380 & 39631 & 127 & 278 & 315 & - & - & - & 42677 \\
\hline & Prevalence \% & 29.41 & 12.02 & 19.46 & 6 & 30.68 & 16 & - & - & - & 19.45 \\
\hline \multirow{3}{*}{ 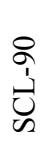 } & $\begin{array}{l}\text { Number of } \\
\text { studies }\end{array}$ & 5 & 1 & 2 & 1 & - & 2 & - & 1 & - & 12 \\
\hline & Sample size & 2262 & 279 & 1100 & 120 & - & 1665 & - & 150 & - & 5576 \\
\hline & Prevalence $\%$ & 15.87 & 10.03 & 9.08 & 32.5 & - & 14.16 & - & 15.62 & - & 13.63 \\
\hline
\end{tabular}

Note. $\mathrm{A}=$ Pregnancy, $\mathrm{B}=$ Postpartum, $\mathrm{C}=$ Postmenstrual, $\mathrm{D}=$ Menstrual

\section{Discussion}

In this study, prevalence of depression was investigated using method of systematic review. A total of 145 studies analyzed had used 15 different tools. The majority of studies had used BDI, which was therefore used as the basis of calculation in this study. Accordingly, overall prevalence of depression was found $42.59 \%$, which is higher than prevalence of depression in general public reported in reference books (15\% to $25 \%)$.

In addition, prevalence of depression in different groups (children, adolescents, students, general public, and the older adults, pregnant and postpartum women) was investigated in detail. Prevalence of depression in school pupils was found $43.55 \%$ with BDI, $15.87 \%$ with SCL-90, and $13.5 \%$ with CDI. Odds of being depressed always exists from childhood to old age, but in most cases onset of symptoms occurs in adolescence or early young age [19]. In a study conducted by Polly et al. in Italy titled "self-reported symptoms of depression in 8-17 year-old adolescents" in $2003,10 \%$ of participants scored higher than 20 (threshold of clinical symptoms) [20]. In Nigeria, the symptom of depression in school pupils was reported 9\% [21], and in Ireland, O'Farrell et al, found severe depression in $20.8 \%$ of school pupils [22]. In a study by Biros, prevalence of moderate to severe depression was reported 20\% [23]. Moreover, prevalence of depression in different sexes of pupils was reported differently using CDI and BDI, but both showed higher prevalence among girls, which agrees with results found by Polly et al. in Italy [20]. Biological, psychological and social differences appear to affect prevalence of depression in two sexes, such that in adolescence, girls are more predisposed to mood disorders and depression [24].

In Iranian studies on prevalence of depression among university students, BDI and GHQ scale have been used, and prevalence of depression is $52.12 \%$ if BDI is taken as the basis. Various studies have reported different prevalence of depression among students in different countries. For instance, prevalence of depression was reported 39\% among university students in Pakistan [25], $23.3 \%$ in undergraduate medical students in Nigeria [26], and $30.6 \%$ in medical students in Estonia [27]. However, despite different statistics, prevalence of depression in these studies is generally lower compared to depression among Iranian students. Of course, the difference may be due to type of tool used, culture, and timing of assessment. Prevalence of depression was found $50.48 \%$ in boys and $48.35 \%$ in girls. Greater future employment and social stresses in boys in Iran can probably be blamed for higher depression in them; whereas, prevalence of depression in girls in nearly all countries and cultures is about twice that in boys. 
Meta-analysis of studies on prevalence of depression in general population showed prevalence of $37.32 \%$ with BDI and $19.46 \%$ with GHQ. If BDI is assumed the basis (with prevalence of $37.32 \%$ ), then prevalence found with BDI is higher than that presented in reference books (15\% to $25 \%)$. With BDI, prevalence of depression is found higher in the female section of the general population, which may be attributed to various factors such as hormonal changes associated with reproductive cycle and mental pressures caused by pregnancy and labor [28].

Review of studies on prevalence of depression in the older adults using GDS scale showed prevalence of $57.58 \%$ in older adults living at home and $81.85 \%$ in older adults living in nursing homes. Clearly, depression is higher in old age compared to that in general population. The present study results are in line with those in a systematic review study by Sajadi et al [29]. Review of prevalence of depression in the older adults in other countries shows different values, for instance, overall prevalence of depression in older adults of $30.3 \%$ in Greece [30], 16.8\% in Netherlands [31], and 4.5\% in Japan [32].

Review of studies on prevalence of depression in pregnant women with BDI showed prevalence of approximately $27.62 \%$. In their systematic review study, Sajadi et al. also found similar prevalence of depression in pregnant women [33]. Pregnancy is a major stress, which can expose or exacerbate dysphoric tendencies. In a study conducted in Malta, prevalence of depression in pregnant women, using Edinburgh scale, was reported $15.5 \%$ [34]. In another study in Paris, using BDI, prevalence of depression in pregnant women was reported $13.9 \%$ [35]. Chan et al, reported prevalence of depression in pregnant women in Singapore $20 \%$ [36].

Synthesis of studies on postpartum depression showed prevalence of $32.52 \%$ with Edinburgh and $28.66 \%$ with BDI. Prevalence of postpartum depression was reported 5.5\% in Denmark [37], 40.4\% in Turkey [38], $17 \%$ in China [39], and $23 \%$ in Nigeria [40]. According to these statistics, postpartum depression is more common in developing compared to developed countries. Perhaps, other factors such as culture and customs are also important along with the issue of socioeconomic development. Generally, huge prevalence of postpartum depression and its serious consequences for mother, infant, and the family beget special attention to this problem.

Study limitations included limited number of English databases. Generally, articles in more well-known databases are more likely to be used in systematic reviews. But this does not necessarily mean higher quality of articles. It is recommended that future studies systematically investigate prevalence of depression in specific periods of time and identify factors affecting different population groups separately, so that appropriate preventive measures can be taken.

\section{Conclusion}

According to the present study results, although differences in methodology, tools, and culture explain differences in prevalence of depression in different cultures, based on statistics provided, prevalence of depression in Iran appears relatively higher than that in other countries. Thus, such a high prevalence of depression in domestic studies, especially in old-age, pregnancy and postpartum periods suggests the profound need for preventive measures and greater attention to this issue.

\section{Acknowledgements}

This research is derived from Approved projects of Social Determinants of Health Research Center. We appreciate the Cooperation by honorable research deputy of university and the head of research center for social determinant of health, and all participants in this study.

\section{Contribution}

Study design: VF, SH MK, HS

Data collection. and analysis: MV, HR, ASF, MM, SHMK

Manuscript preparation: MM 


\section{Conflict of Interest}

"The authors declare that they have no competing interests."

\section{Funding}

The authors received financial support (code: 91/801/ T/7364) for the research from department of social determinants of health of university of social welfare and rehabilitation science.

\section{References}

1- Patel V, Kleinman A. Poverty and common mental disorders in developing countries. Bull World Health Organ2003; 81(8): 609-15.

2- American psychiatric association. The diagnostic and statistical manual of mental disorders. 4 editor. Washington, DC: American psychiatric association; 2000 .

3- Lambert KG. Rising rates of depression in today's society: consideration of the roles of effort-based rewards and enhanced resilience in day-to-day functioning. Neuroscience and Biobehavioral2006; (30): 497- 510.

4- Sadeghi M, Kazemi H. Prevalence of dementia and depression among residents of elderly nursing homes in Tehran province. Andeesheh Va Raftar2003; 9(36): 4955.

5- Noorbala A, Mohammad K, Bagheri-Yazdi S, Yasami M. Study of mental health status of individuals above 15 years of age in Islamic Republic of Iran in the year 1999. Hakim 2001; 5(1): 1-10.

6- Mohammadi M, Davidian H, Noorbala A, et al. An epidemiological study of psychiatric disorders in Iran, 2001. Hakim 2002; 6(1): 55-64.

7- Montazeri A, Mousavi SJ, Omidvari S, Tavousi M, Hashemi A, Rostami T. Depression in Iran: a systematic review of the literature (2000-2010). payesh2013; 12(6). 8- Sarukhni D, Visani Y, Sarukhani M, Esmaili R. Prevalence of depression in Iranian students: a systematic review and meta-analysis. Research in Medicine2012; 36(1).

9- Sadeghirad B, Haghdoost AA, Amin Esmaeili M, et al. Epidemiology of major depressive disorder in Iran: a systematic review and meta-analysis. Int J Prev Med2010; 1(2): 81.

10- Vameghi M. Systematic review of studies on street children in Iran in recent decade. Social Welfare Quarterly2009; 9(35): 337-78.

11- Khan SR, Kleignen J, Antes G. Systematic reviews to support evidence based medicine: how to review and apply findings of healthcare research. London: Royal society of medicine press ltd; 2003.
12- Rahimikian F, Amelvalizadeh M, Ghobari Bonab B, Mehran A, Rahimi N. Frequency and intensity of depression during menstrual period and It's relation with religious attitudes in Karaj high school girls 2003. Dena, Yasuj Faculty of Nursing And Midwifery2005; 1(2): 1-9.

13- Zarrabi H, Koosha M, Amiri ZM, Kiani S. Prevalence of psychopathology in high school students in Rasht. J Medical Faculty Guilan university of Medical Sciences2000; 10(40-39): 55-0.

14- Jenaabadi H, Nastiezaie N. A comparison of aggression, anxiety and depression in high school students in saravan city. Urmia Nursing And Midwifery Faculty2011; 9(3): 148-56.

15- Sepehrmanesh Z, Ahmadvand A, Yavari P, Saei R. Assessing the mental health of adolescents in Kashan, 2004. Iranian Journal of Epidemiology2008; 4(2): 43-9. 16- Hosseini Fard SM, Birashk B, Atef Vahid MK. Epidemiology of mental disorders in high-school students in Rafsanjan. Andeesheh Va Raftar2005; 11(40): 71-80.

17- Hosseini SH, Mousavi S, Rezazadeh H. Assessment of mental health in students in Sari, 2001-02. Quarterly Journal of Fundamentals of Mental Health2003; 6(19): 92-9.

18- Motaghi Pour Y, Valaei F, Amiri P, et al. Study of the general mental health status in an area of Tehran: Tehran Lipid and Glucose Study (TLGS). Iranian Journal of Endocrinology \& Metabolism2004; 7(28): 301-6.

19- scully I. Psychiatry. 20, editor. Philadelphia: Baltimor williams \& wilkins; 1995.

20- Poli P, Sbrana B, Marcheschi M, Masi G. Selfreported depressive symptoms in a school sample of Italian children and adolescents. Child Psychiatry Hum Dev2003; 33(3): 209-26.

21- Adewuya AO, Ologun YA. Factors associated with depressive symptoms in nigerian adolescents. $J$ Adolesc Health2006; 39(1): 105-10.

22- O'Farrell A, Flanagan E, Bedford D, James D, Howell F. Factors associated with self-reported depression and self-esteem among school-going adolescents from a geographically defined region in Ireland. Iranian Journal of Medical Sciences,2005; 174(4): 17-22.

23- Biros MH, Hick K, Cen Y, et al. Occult depressive symptoms in adolescent emergency department patients. Archives of Pediatrics \& Adolescent Medicine2008; 162(8): 769-73.

24- No authors listed. Practice parameters for the assessment and treatment of children and adolescents with depressive disorders. AACAP. J Am Acad Child Adolesc Psychiatry1998; 37(10): 63-83.

25- Zaman RM. Psychological problems of medical 
students in pakistan: data from the aga khan university, Karachi. Teaching and Learning in Medicine 1996; 8(1): 19-27.

26- Aniebue PN, Onyema GO. Prevalence of depressive symptoms among nigerian medical undergraduates. Trop Doct 2008; 38(3): 157-8.

27- Eller T, Aluoja A, Vasar V, Veldi M. Symptoms of anxiety and depression in Estonian medical students with sleep problems. Depress Anxiety2006; 23(4): 250-6.

28- Bloch M, Rotenberg N, Koren D, Klein E. Risk factors for early postpartum depressive symptoms. Gen Hosp Psychiatry2006; 28(1): 3-8

29- Sajadi H, Mohaqeqi Kamal H, Vameghi M, Forozan AS, Rafei H, Nosratabadi M. Systematic review of prevalence and risk factors associated with depression and its treatment in Iranian elderly. Iranian Journal of Ageing2013; 7(27): 7-15.

30- Carayanni V, Stylianopoulou C, Koulierakis G, Babatsikou F, Koutis C. Sex differences in depression among older adults: are older women more vulnerable than men in social risk factors? The case of open care centers for older people in Greece. Eur J Ageing2012; 9(2): 177-86.

31- Hermans H, Beekman AT, Evenhuis HM. Prevalence of depression and anxiety in older users of formal dutch intellectual disability services. J Affect Disord2013; 144(1-2): 94-100.

32- Hidaka S, Ikejima C, Kodama C, et al. Prevalence of depression and depressive symptoms among older Japanese people: comorbidity of mild cognitive impairment and depression. Int J Geriatr Psychiatry2012; 27(3): 271-9.

33- Sajadi H, Vameghi M, Setare forozan A, Rafiey H, Mohgheghee Kamal S, Nosrat abadi M. Prevalence, risk factors and interventions of depression during pregnancy in Iranian Studies (1997-2011): A systematic review. $J$ North Khorasan University of Medical Sciences2013; 5(2): 521-30.

34- Felice E, Saliba J, Grech V, Cox J. Prevalence rates and psychosocial characteristics associated with depression in pregnancy and postpartum in Maltese women. $J$ Affect Disord2004; 82(2): 297-301.

35- LteifY, Kesrouani A, Richa S. Depressive syndromes during pregnancy: prevalence and risk factors. $J$ Gynecol Obstet Biol Reprod (Paris)2005; 34(3 Pt 1): 262-9.

36- Chen H, Chan YH 3rd, Tan KH, Lee T. Depressive symptomatology in pregnancy-a Singaporean perspective. Soc Psychiatry Psychiatr Epidemiol2004; 39(12): 975-9.

37- Nielsen Forman D, Videbech P, Hedegaard M, Dalby Salvig J, Secher NJ. Postpartum depression: identification of women at risk. BJOG2000; 107(10): 1210-7.

38- Ekuklu G, Tokuc B, Eskiocak M, Berberoglu U, Saltik A. Prevalence of postpartum depression in edirne,
Turkey, and related factors. $J$ Reprod Med2004; 49(11): 908-14.

39- Xie RH, He G, Liu A, Bradwejn J, Walker M, Wen SW. Fetal gender and postpartum depression in a cohort of Chinese women. Social Science \& Medicine2007; 65(4): 680-4.

40- Owoeye AO, Aina OF, Morakinyo O. Risk factors of postpartum depression and EPDS scores in a group of Nigerian women. Trop Doct2006; 36(2): 100-3. 